\title{
Lentiviral RNA interference-mediated downregulation of Forkhead box M1 expression suppresses growth of oral squamous cell carcinoma in vitro
}

\author{
JING QIU $^{1}$, JUAN ZHAO ${ }^{2}$, ANJUN ZUO $^{3}$, LAN LIU $^{1}$, QIAOQIAO LIU ${ }^{1}$, HUAZHENG PAN $^{4}$ and XIAO YUAN $^{1}$ \\ ${ }^{1}$ Department of Stomatology, Qingdao Municipal Hospital, Qingdao, Shandong 266071; \\ ${ }^{2}$ Department of Pediatrics, Jiaozhou People's Hospital, Qingdao, Shandong 266300; ${ }^{3}$ Medical Services; \\ ${ }^{4}$ Clinical Laboratory, The Affiliated Hospital of Qingdao University, Qingdao, Shandong 266003, P.R. China
}

Received April 6, 2016; Accepted May 10, 2018

DOI: $10.3892 / \mathrm{ol} .2018 .9536$

\begin{abstract}
Oral squamous cell carcinoma (OSCC) is one of the most fatal types of oral cancer worldwide. Forkhead box M1 (FOXM1) is associated with the occurrence and development of a number of types of human cancer, but its function in OSCC remains unclear. The present study aimed to explore the effect of FOXM1 downregulation using lentivirus-mediated short hairpin (sh)RNA against FOXM1 (LV-shFOXM1) in the cell line Tca8113 in vitro. Infection of Tca8113 cells with LV-shFOXM1 inhibited the mRNA and protein expression level of FOXM1. The downregulation of FOXM1 resulted in cell cycle arrest of Tca8113 cells, and the inhibition of proliferation, migration and invasion. The protein expression level of cyclins B1 and D1 were downregulated, whereas those of p27 and p21 were upregulated following infection with LV-shFOXM1, compared with the blank control and LV-shCON groups. In addition, FOXM1 downregulation decreased the expression of matrix metalloproteinase- 2 and LV-shFOXM1 significantly suppressed OSCC cell viability. Therefore, FOXM1 may be a target for the treatment of OSCC.
\end{abstract}

\section{Introduction}

Oral squamous cell carcinoma (OSCC), one of the most fatal types of cancer (1), is identified with frequent lymph node and distant metastases (2). Metastasis is a key prognostic

Correspondence to: Dr Huazheng Pan, Clinical Laboratory, The Affiliated Hospital of Qingdao University, 16 Jiangsu Road, Qingdao, Shandong 266003, P.R. China

E-mail: hzpanmed18@126.com

Dr Xiao Yuan, Department of Stomatology, Qingdao Municipal Hospital, 5 Donghai Middle Road, Qingdao, Shandong 266071, P.R. China

E-mail: xyuan208@126.com

Key words: oral squamous cell carcinoma, Forkhead box M1, growth inhibition factor for OSCC and typically indicates a poor prognosis (3). However, the underlying molecular mechanisms that regulate metastatic dissemination remain unclear, despite a number of studies on the molecular mechanisms implicated in OSCC development (4).

Forkhead box M1 (FOXM1), which belongs to the Fox family of transcription factors, maintains a balance between proliferation and apoptosis of cells (5). Mutation of FOXM1 prevents differentiation, resulting in the malignant transformation of undifferentiated cells (6). Overexpression of FOXM1 is associated with the occurrence and development of numerous cancer types (7). FOXM1 is an essential cell cycle regulator in the $G_{1} / S$ and $G_{2} / M$ stage transitions and mitosis (8), and thus contributes to cell proliferation. Furthermore, FOXM1 is important in tumor angiogenesis, epithelial-mesenchymal transition, invasion and metastasis (9). Silencing of FOXM1 inhibits the proliferation, invasion and migration of human colorectal cancer (CRC) cells (10). However, the precise functions and underlying molecular mechanisms of FOXM1 in OSCC cells remain unclear.

In the present study, the expression level of FOXM1 was determined in OSCC cells, and the effect of FOXM1 gene knockdown on the proliferation, migration and invasion of Tca8113 cells was investigated in vitro. The results of the present study identified that the downregulation of FOXM1 suppressed the activities of Tca8113 cells, and decreased the expression of proteins associated with cell cycle and viability. These results have suggested that FOXM1 is a therapeutic target for the treatment of OSCC.

\section{Materials and methods}

Cell culture reagents. Human HaCaT, Tca8113 and SCC9 cells were purchased from the American Type Culture Collection (Manassas, VA, USA). SCC9 cells were kept in a 1:1 mixture of Dulbecco's modified Eagle's medium (DMEM) and Ham's F12 medium supplemented with $10 \%$ fetal bovine serum (FBS), $1 \%$ penicillin/streptomycin solution (all from Invitrogen; Thermo Fisher Scientific, Inc., Waltham, MA, USA), and $400 \mathrm{ng} / \mathrm{ml}$ hydrocortisone (Sigma-Aldrich; Merck KGaA, Darmstadt, Germany). HaCaT and Tca8113 
cells were incubated at $37^{\circ} \mathrm{C}$ in an atmosphere containing $5 \% \mathrm{CO}_{2}$ with saturated humidity and cultured as monolayers in RPMI-1640 medium (Gibco; Thermo Fisher Scientific, Inc.) supplemented with $10 \%$ FBS, $2 \mathrm{mM}$ glutamine and $1 \%$ penicillin/streptomycin. Trypsin-ethylenediaminetetra acetic acid (Invitrogen; Thermo Fisher Scientific, Inc.) was used to detach cells.

Reverse transcription-quantitative polymerase chain reaction (RT-qPCR). Total RNA from all cell lines was isolated using TRIzol reagent (Invitrogen; Thermo Fisher Scientific, Inc.) and reverse transcribed into cDNA using $5 \mathrm{X}$ PrimeScript RT Master mix (Takara Bio, Inc., Otsu, Japan) at $37^{\circ} \mathrm{C}$ for $15 \mathrm{~min}$ and $85^{\circ} \mathrm{C}$ for $5 \mathrm{sec}$, according to the manufacturer's protocol. Gene expression levels were determined using 2X SYBR Premix Ex Taq (Takara Bio, Inc.) with a 7300 ABI RT-PCR system (Applied Biosystems; Thermo Fisher Scientific, Inc.) for 40 cycles at $95^{\circ} \mathrm{C}$ for $30 \mathrm{sec}, 95^{\circ} \mathrm{C}$ for $5 \mathrm{sec}$ and $60^{\circ} \mathrm{C}$ for $31 \mathrm{sec}$. The relative mRNA levels were determined using the $2^{-\Delta \Delta c q}$ method and normalized to the expression of GAPDH (11). The sequence of the primers were as follows: FOXM1, forward, 5'-ATACGTGGATTGAGGACCACT-3' and reverse, 5'-TCC AATGTCAAGTAGCGGTTG-3'; GAPDH forward, 5'-ACT GCCACCCAGAAGACT-3' and reverse, 5'-GCTCAGTGT AGCCCAGGAT-3'.

Design of FOXM1 RNA interference (RNAi) sequence and construction of short hairpin (sh)RNA-expressing lentiviral vectors. A total of three FOXM1-specific sequences were selected using the online short interfering RNA tools (Invitrogen; Thermo Fisher Scientific, Inc.; www.invitrogen. com/rnai) with the reference sequence of FOXM1 (gene bank accession no. NM_021953). The target sequences of FOXM1-1 (5'-TTGCAGGGTGGTCCGTGTAAA-3'), FOXM1-2 (5'-TTG CAGGGTGGTCCGTGTAAA-3') and FOXM1-3 (5'-AGG ACCACTTTCCCTACTTTA-3') were homologous with those of FOXM1-specific mRNA. The transfection of siRNA oligonucleotides was performed with Lipofectamine2000 (Invitrogen; Thermo Fisher Scientific, Inc.), according to the manufacturer's protocols. Briefly, $16 \mu 1$ of Lipofectamine 2000 reagent was mixed with $400 \mu$ l of Opti-MEM (Invitrogen; Thermo Fisher Scientific, Inc.) at room temperature for $5 \mathrm{~min}$ and subsequently incubated with a mixture of $12 \mu \mathrm{l}$ of $20 \mu \mathrm{M}$ siRNA duplex and $400 \mu \mathrm{l}$ of Opti-MEM for an additional $20 \mathrm{~min}$ at room temperature. The complexes were then applied to cultured cells at $\sim 70 \%$ confluency on a $60-\mathrm{mm}$ plate containing $4 \mathrm{ml}$ of RPMI-1640 medium. After $12 \mathrm{~h}$ incubation, the medium was replaced with fresh RPMI-1640 medium supplemented with $10 \%$ fetal bovine serum and $1 \%$ penicillin/streptomycin. The chemical synthesis of shRNA lentivirus vector construction was performed according to a previous study (12). The lentiviral shRNA was constructed harboring green fluorescent protein. The invalid RNAi sequence (5'-GAGCTATGGCAGCTACCATCA-3') was used as a negative control. The appropriate insertion of the specific shRNA was validated by sequencing.

Cell extracts and western blot analysis. Protein lysates were prepared on ice using a radioimmunoprecipitation assay buffer [150 mM NaCl, 50 mM Tris-HCl (pH 8.0), 1\% NP40,
$0.1 \%$ SDS and $0.5 \%$ sodium deoxycholate] supplemented with $1 \mathrm{mg} / \mathrm{ml}$ aprotinin, $1 \mathrm{mM}$ sodium orthovanadate and $0.1 \mathrm{mg} / \mathrm{ml}$ phenylmethylsulfonyl fluoride. Protein contents were determined using a bicinchoninic acid protein assay system (Bio-Rad Laboratories Inc., Hercules, CA, USA). Equal amounts of cell extracts containing between 20 and $50 \mu \mathrm{g}$ of total protein were separated using $12 \%$ SDS-PAGE and transferred to $0.45-\mu \mathrm{m}$ nitrocellulose membranes (Osmonics, Westborough, MA, USA). The membranes were blocked for $1 \mathrm{~h}$ in Blotto A (Beyotime Institute of Biotechnology, Shanghai, China) at room temperature which consisted of $5 \%$ non-fat milk powder in Tris-buffered saline and $0.05 \%$ Tween-20 (TBS-T), containing $10 \mathrm{mM}$ Tris- $\mathrm{HCl}$ (pH 8.0) and $150 \mathrm{mM} \mathrm{NaCl}$. Subsequently, membranes were incubated for $1 \mathrm{~h}$ at room temperature in Blotto A containing a 1:1,000 dilution of the following rabbit primary antibodies: Anti-FOXM1 (cat. no. 5436; Cell Signaling Technology, Danvers, MA, USA), anti-GAPDH (cat. no. ab8245; Abcam, Cambridge, MA, USA), anti-cyclin B1 (cat. no. 12231), anti-cyclin D1 (cat. no. 2926), anti-P27 (cat. no. 3686), anti-P21 (cat. no. 2947) and anti-matrix metalloproteinase (MMP) 2 (cat. no. 40994) (all from Cell Signaling Technology Inc.). Membranes were washed in TBS-T for $5 \mathrm{~min}$ and incubated for $1 \mathrm{~h}$ at room temperature in Blotto A containing a 1:10,000 dilution of peroxidase-conjugated anti-rabbit (cat. no. RPN4301) or anti-mouse secondary antibody (cat. no. RPN4201) (GE Healthcare, Chicago, IL, USA). Following washing in TBS-T, enhanced chemiluminescence was performed according to the manufacturer's protocol.

MTT assay. The cytotoxic activity of LV-shFOXM1 was determined on the basis of cytotoxicity to Tca8113 cells using an MTT assay. Cells were seeded in 96-well plates $\left(5 \times 10^{3}\right.$ cells/well) and treated with $\mathrm{LV}$-shCON (control) or LV-shFOXM1. The following day, $100 \mu 1$ fresh RPMI-1640 medium was added to each well with $0.5 \mathrm{mg} / \mathrm{ml}$ MTT (Roche Applied Science, Manheim, Germany). After $4 \mathrm{~h}$ of incubation at $37^{\circ} \mathrm{C}$ in a humidified atmosphere containing $5 \% \mathrm{CO}_{2}$, $150 \mu \mathrm{l}$ solubilization solution $(0.01 \mathrm{~mol} / 1 \mathrm{HCl}$ in $100 \mathrm{~g} / \mathrm{l}$ SDS) was added. Subsequently, cells were gently agitated for $10 \mathrm{~min}$ at $37^{\circ} \mathrm{C}$. The absorbance was determined at $450 \mathrm{~nm}$ on microplate reader (model 550; Bio-Rad Laboratories, Inc.) as the optical density of the plates. Cell viability was evaluated for four consecutive days. Each MTT assay was performed in triplicate.

Cell cycle analysis via flow cytometry. Tca8113 cells, at the logarithmic phase, were seeded in 6-well plates $\left(6 \times 10^{5}\right.$ cells/well $)$ and incubated at $37^{\circ} \mathrm{C}$ in humid conditions containing $5 \% \mathrm{CO}_{2}(\mathrm{v} / \mathrm{v})$ for $24 \mathrm{~h}$, followed by $24 \mathrm{~h}$ of treatment with LV-shFOXM1 or LV-shCON in RPMI-1640. Subsequently, the cells were washed with ice-cold PBS twice and fixed in ice-cold $70 \%(\mathrm{v} / \mathrm{v})$ ethanol at $-20^{\circ} \mathrm{C}$ overnight. Following treatment with $10 \mathrm{mM}$ Tris- $\mathrm{HCl}$ buffer $(\mathrm{pH} 7.5)$ with $1 \%$ (w/v) RNase A (Sigma-Aldrich; Merck KGaA) for $15 \mathrm{~min}$ at $4^{\circ} \mathrm{C}$ the cells were incubated with propidium iodide (Sigma-Aldrich; Merck KGaA) for $15 \mathrm{~min}$ at $4^{\circ} \mathrm{C}$. The cell cycle was analyzed using a flow cytometer (BD Biosciences, Franklin Lakes, NJ, USA), and the data were analyzed using ModFit LT 2.0 (Verity Software House, Topsham, ME, USA). 
A

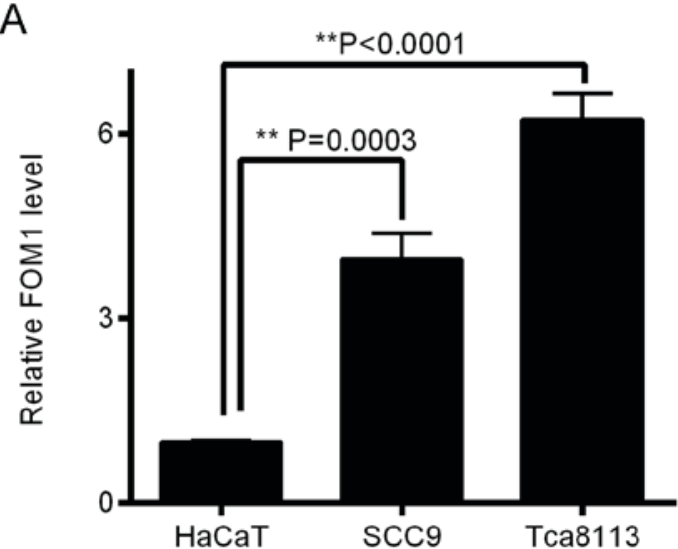

B

\begin{tabular}{|ll} 
HaCaT $\quad$ SCC9 & Tca8113 \\
\hline & \\
\hline & GOXM1 \\
& GAPDH
\end{tabular}

Figure 1. Expression level of FOXM1 in HaCaT and human OSCC cells (SCC9 and Tca113) determined using (A) reverse transcription-quantitative polymerase chain reaction and (B) western blot analysis. GADPH was used as the loading control. Experiments were performed in triplicate. ${ }^{* *} \mathrm{P}<0.0001 \mathrm{vs}$. HaCaT. Forkhead box M1; OSCC, oral squamous cell carcinoma.

Soft agar assays. The aim of this assay was to analyze the effect of FOXM1-shRNA on the proliferation of Tca8113 cells. Briefly, cells were subjected to soft agar assay in 6-well plates and 50,000 cells/well were added into each well, which consisted of a bottom base layer (0.6\% agar diluted in DMEM with $10 \%$ FBS) and top layer (0.3\% agarose diluted in DMEM with $10 \% \mathrm{FBS})$. The cells were cultured at $37^{\circ} \mathrm{C}$ for 2 weeks. Colonies were scored following 2 weeks of cell incubation. Each protocol was repeated at least three times.

Cell migration and invasion assays. The assays were conducted using Transwell chambers equipped with a pore size of $8 \mu \mathrm{m}$, according to the manufacturer's protocol. After $24 \mathrm{~h}$ of infection, Tca8113 cells were resuspended in serum-free DMEM $\left(2 \times 10^{4}\right.$ cells/well) and seeded into the Transwell inserts either uncoated (migration assay) or coated with growth factor-reduced Matrigel (BD Biosciences) (invasion assay). The lower chambers were filled with $500 \mu 1$ DMEM containing $10 \% \mathrm{FBS}$. After $24 \mathrm{~h}$ of incubation at $37^{\circ} \mathrm{C}$, all cells on the upper side of the insert filter were selected using a cotton swab, while the invaded cells were $100 \%$ methanol-fixed for $20 \mathrm{~min}$ at room temperature and stained with $0.1 \%$ crystal violet for $10 \mathrm{~min}$ at room temperature. The manual cell counting was finished under an inverted microscope (Olympus IX51; Olympus Corporation, Tokyo, Japan) on five random fields (magnification, 40x; scale bar, $200 \mu \mathrm{m}$ ). Each protocol was performed in triplicate.

Statistical analysis. Data are expressed as the mean \pm standard deviation. Significant differences between groups were examined using the Student's t-test and the $\chi^{2}$ test. $\mathrm{P}<0.05$ was considered to indicate a statistically significant difference. All statistical tests were conducted using SPSS software (version 17.0; SPSS, Inc., Chicago, IL, USA).

\section{Results}

FOXM1 mRNA is expressed at an increased level in human OSCC. FOXM1 overexpression has been identified in human head and neck squamous cell cancer, ovarian cancer and hepatoma (13), but has rarely been demonstrated in OSCC cells. In the present study, RT-qPCR revealed a significant overexpression of FOXM1 mRNA in OSCC cells compared with $\mathrm{HaCaT}$ cells $(\mathrm{P}<0.01$; Fig. $1 \mathrm{~A})$. To validate these results, western blot analysis was conducted to determine FOXM1 protein expression. The results demonstrated that FOXM1 protein was markedly overexpressed in OSCC cells compared with $\mathrm{HaCaT}$ cells (Fig. 1B). In the present study, RT-qPCR and western blot analysis validated the expression of FOXM1 in OSCC cell lines compared with human immortal keratinocyte cell line HaCaT (Fig. 1A and B), and indicated that Tca8113 was an appropriate cell line for RNAi targeting FOXM1 mRNA.

LV-shFOXM1-2 is the optimal vector. Tca8113 cells were infected with four plasmids separately for $48 \mathrm{~h}$, including shFOXM1 (LV-shFOXM1-1, -2 and -3) and LV-shCON. Subsequently, the expression of green fluorescent protein in Tca8113 cells was observed under a fluorescent microscope (Fig. 2A). RT-qPCR revealed that LV-shFOXM1-2 significantly inhibited the expression of FOXM1 mRNA compared with the shCON and shFOXM1 groups (Fig. 2B; $\mathrm{P}<0.0001$ ). Western blot analysis demonstrated that LV-shFOXM1-2 was the optimal vector and therefore was selected for use in subsequent protocols (Fig. 2C), and termed LV-shFOXM1.

Downregulation of FOXM1, using LV-shFOXM1, suppresses cell growth, colony formation and cell cycle at the $S$-phase. FOXM1 is a key regulator of animal growth and cell proliferation (13). The downregulation of FOXM1 expression in Tca8113 cells significantly inhibited cell proliferation, compared with the blank and LV-shCON groups $(\mathrm{P}<0.05$; Fig. $3 \mathrm{~A})$. The association between the downregulation of FOXM1 expression and alterations in cycle progression was analyzed in Tca8113 cells by the flow cytometer. The proportion of S-phase cells following LV-shFOXM1 infection was significantly decreased compared with the blank and LV-shCON groups $(\mathrm{P}<0.05$; Fig. 3B). Subsequently, whether FOXM1 gene knockdown decreases the colony formation of Tca8113 cells was investigated. After $24 \mathrm{~h}$ of infection with LV-shFOXM1, Tca8113 

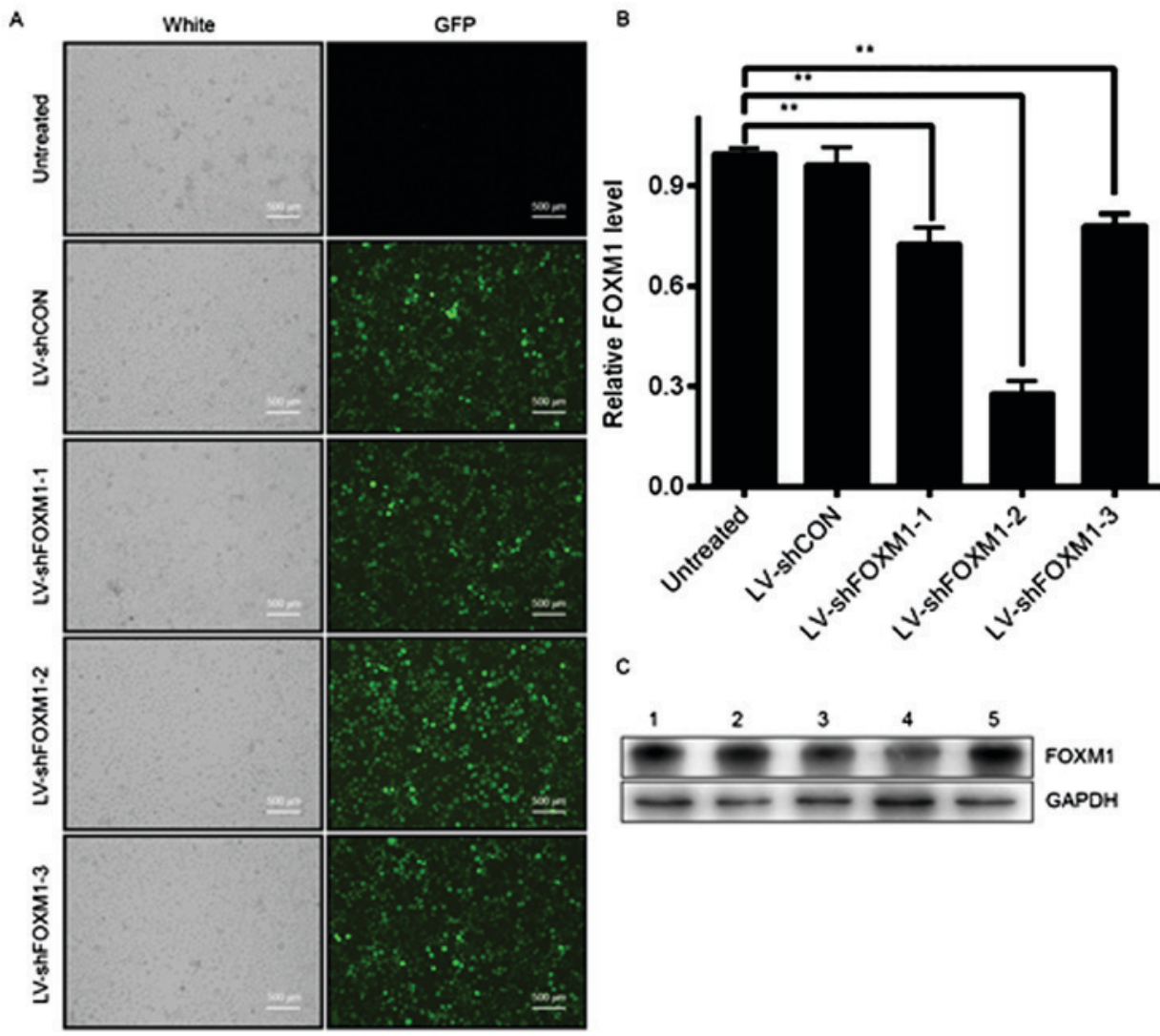

Figure 2. Selection of the optimal LV-shFOXM1 in Tca8113 cells. (A) GFP expression in Tca8113 cells after $48 \mathrm{~h}$ of infection, determined under a fluorescence microscope. (B) mRNA level of FOXM1 following lentiviral infection, determined using reverse transcription-quantitative polymerase chain reaction (C) Protein level of FOXM1 determined using western blot analysis. GADPH was used as the loading control. Lane 1, Tca8113 cells were not infected; lane 2, Tca8113 cells were infected with LV-shCON; lane 3, Tca8113 cells were infected with LV-shFOXM1-1; lane 4, Tca8113 cells were infected with LV-shFOXM1-2; and lane 5, Tca8113 cells were infected with LV-shFOXM1-3. ${ }^{* *} \mathrm{P}<0.01$ vs. untreated. LV-shFOXM1, lentivirus-short hairpin RNA Forkhead box M1; CON, control; GFP, green fluorescent protein.

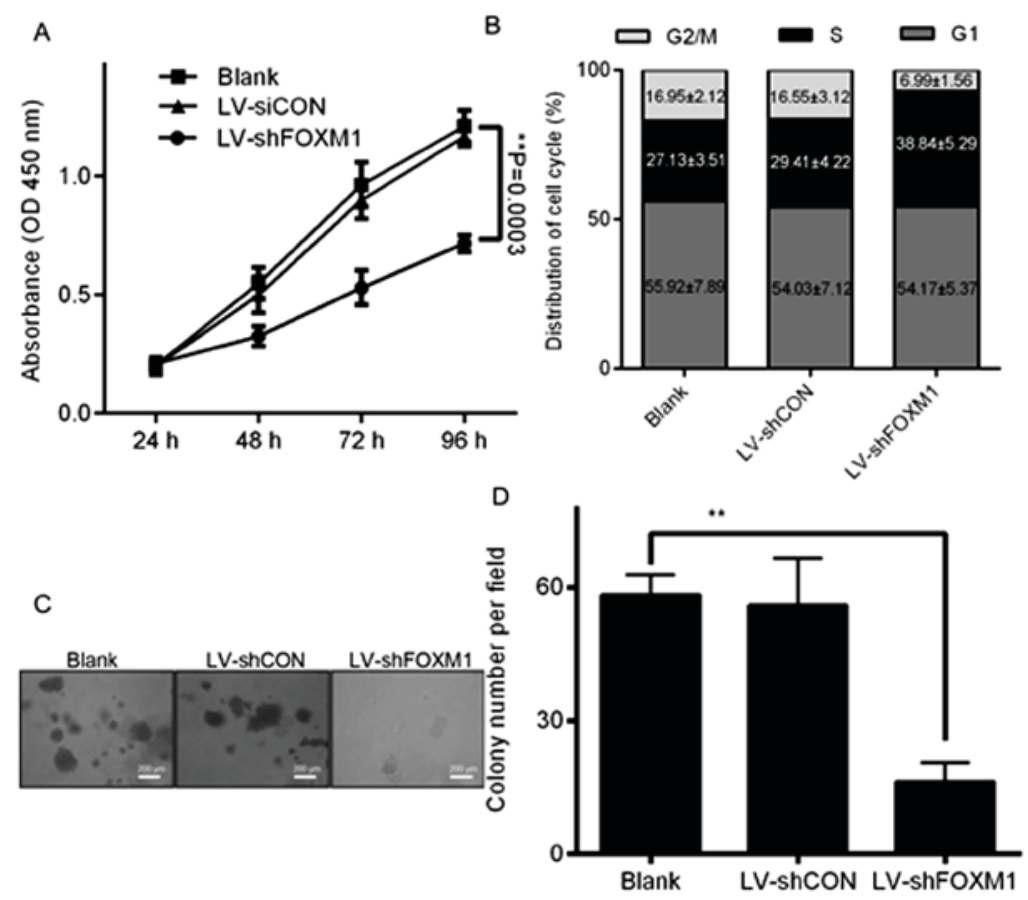

Figure 3. Downregulation of FOXM1 affects proliferation, cell cycle and clonogenicity of Tca8113 cells. (A) MTT assay revealed the inhibition of viability of Tca8113 cells in LV-shFOXM1 cells, compared with the blank and LV-shCON groups. Tca8113 cell viability was determined following infection for $24,48,72$ and 96 h. (B) S phase arrest was determined using flow cytometry. (C) Representative images of the colony formation assay and (D) quantification of colony formation demonstrating a significant decrease in the number of colonies formed in Tca8113 cells compared with LV-shCON and untreated cells. ${ }^{* *} \mathrm{P}<0.01$ vs. blank. LV-shFOXM1, lentivirus-short hairpin RNA Forkhead box M1; CON, control; OD, optical density. 
A

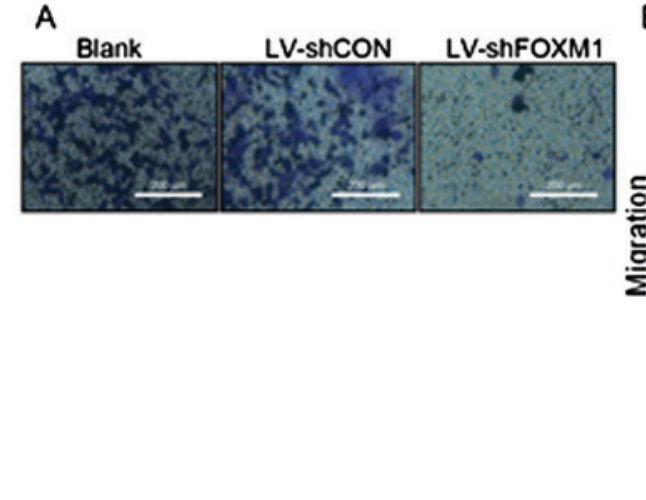

C

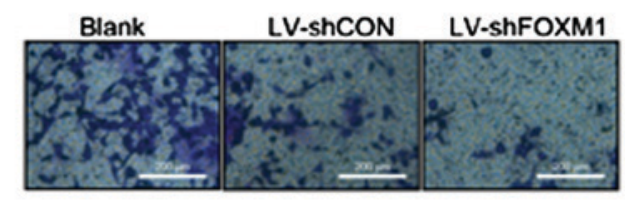

B
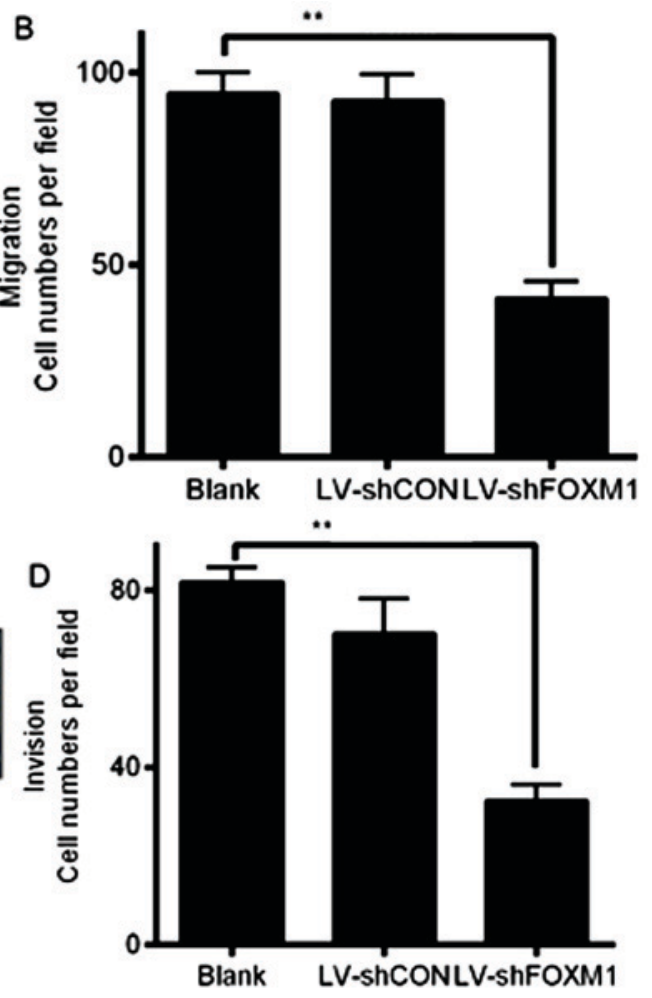

Figure 4. Downregulation of FOXM1 decreases the migratory and invasive abilities of Tca8113 cells. (A) Representative image and (B) quantification of the Transwell migration assay. (C) Representative image and (D) quantification of the Transwell invasion assay. ${ }^{* *} \mathrm{P}<0.01$ vs. blank. LV-shFOXM1, lentivirus-short hairpin RNA Forkhead box M1; CON, control.

cells were seeded in 6-well plates and cultured for 14 days. The results revealed that the number of colonies in LV-shFOXM1 Tca8113 cells were significantly decreased, compared with that of the LV-shCON and untreated cells (Fig. 3C and D). The results of the present study indicated that the downregulation of FOXM1 expression may inhibit cell cycle progression, growth and colony formation of Tca8113 cells.

Downregulation of FOXM1 expression inhibits cell migration and invasion. Cell migration and invasion assays were performed using a Transwell system to determine whether the downregulation of FOXM1 expression may affect the migratory and invasive abilities of Tca8113 cells. The number of cells that migrated or invaded the bottom of the well decreased significantly compard with the blank and LV-shCON groups $(\mathrm{P}<0.05$; Fig. 4). The results indicated that the downregulation of FOXM1, following LV-shFOXM1 infection, inhibited the migratory and invasive abilities of Tca8113 cells, compared with the blank and LV-shCON-infected cells. In addition, western blot analysis revealed that the downregulation of the FOXM1 expression altered expression of proteins associated with cell cycle. As presented in Fig. 5, the expression levels of cyclin B1, cyclin D1 and MMP-2 markedly decreased, but the expression of p27 and p21 markedly increased (LV-shFOXM1 vs. blank and LV-shCON groups).

\section{Discussion}

The overexpression of FOXM1 expression or activity has been associated with the occurrence and development of numerous types of cancer (14-17). Activation of FOXM1, as

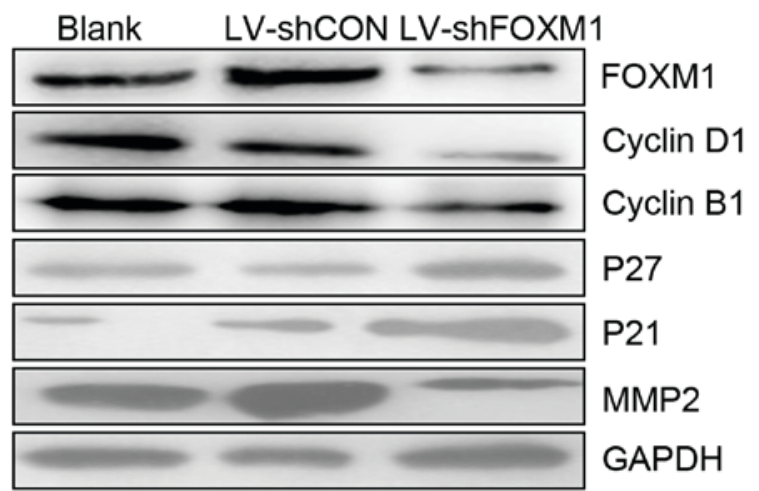

Figure 5. Downregulation of FOXM1 alters the expression levels of proteins associated with the cell cycle, migration and invasion. GAPDH was used as a loading control. LV-shFOXM1, lentivirus-short hairpin RNA Forkhead box M1; CON, control; MMP2, matrix metallopeptidase 2.

an independent poor prognostic factor, is associated with the proliferation and metastasis of human colon cancer cells, and decreased overall survival and metastasis-free survival rates in patients with CRC (18). However, the function of FOXM1 in OSCC remains unclear. In the present study, the mRNA and protein expression level of FOXM1 in OSCC cells was determined using RT-qPCR and western blot analysis, respectively, and was identified to be expressed at increased levels in OSCC cells compared with the human immortal keratinocyte cell line, $\mathrm{HaCaT}$. The results suggested that FOXM1 may be a novel factor in OSCC development.

RNAi-mediated gene silencing is used as the therapy for a variety of diseases (19-21). Lentivirus vectors are considered 
to be a vehicle for efficient gene delivery in research and gene therapy, due to their ability to transform dividing and non-dividing cells, provide stable transgene expression and exhibit decreased toxicity (22). In the present study, a recombinant shRNA-expressing lentiviral vector was constructed, and its effects on the growth and invasion of OSCC cells were observed. Lentivirus-mediated RNAi, which specifically downregulated FOXM1, was identified to be a possible effective treatment for OSCC, as OSCC cells exhibited decreased growth and invasion following the silencing of FOXM1.

FOXM1 has been identified to be a key regulator of the $\mathrm{G}_{1} / \mathrm{S}$ and $\mathrm{G}_{2} / \mathrm{M}$ stage transitions during the cell cycle and is required for proliferative expansion during tumor development (23). Cell proliferation, mediated by cell cycle machinery, depends on the equilibrium between cyclin-dependent kinase (CDK) regulators, in particular $\mathrm{CDK}$ inhibitors (CDKI) and positive factors (cyclins) (24). In the present study, cyclins B1 and D1, and CDKIs (p21 and p27) were selected. The downregulation of FOXM1 expression, using LV-shFOXM1, markedly decreased the protein expression levels of cyclins B1 and D1, and increased that of p27 and p21. The results of the present study indicated that FOXM1 affected the cycle of Tca8113 cells by mediating the expression levels of a number of cyclins and CDKIs. A previous study suggested that silencing the expression of FOXM1 may serve a role in the suppression of proliferation (25).

Cell migratory and invasive abilities are key to cancer invasion and metastasis, which involves the breakdown of extracellular matrix and basement membranes, enabling tumor cells to migrate. Proteolytic enzymes and MMPs have been identified to be involved in cancer invasion and metastasis $(26,27)$. The results of the present study identified that downregulation of FOXM1 expression by LV-shFOXM1 significantly decreased the migratory and invasive abilities of Tca8113 cells. In addition, the expression of MMP2 was inhibited by the downregulation of FOXM1 expression. Therefore, the loss of invasive abilities of OSCC cells, following silencing of FOXM1, may be induced partially by the inhibition of MMP-2.

The results of the present study demonstrated that the downregulation of FOXM1 expression by FOXM1-specific shRNA decreased cell proliferation and the invasive abilities of Tca8113 cells, resulted in the downregulation of cyclins B1, D1 and MMP-2, and upregulated p27 and p21 expression. The results of the present study identified FOXM1 as a functionally important component in the occurrence and development of OSCC, and may be a novel target for the treatment of OSCC.

\section{Acknowledgements}

The authors would like to thank Dr. Shihai Liu, Medical Animal Lab, the Affiliated Hospital of Qingdao University (Qingdao, China) for assisting with the statistical analysis.

\section{Funding}

The present study was supported by Qingdao postdoctoral application research funded project (grant no. 2014011218; Qingdao, China).

\section{Availability of data and materials}

All data generated or analyzed during the present study are included in this published article.

\section{Authors' contributions}

JQ performed signaling pathway analysis, RT-qPCR and wrote the manuscript. QL, JZ and LL performed the migration and invasion experiments. AZ and HP carried out the soft agar analysis. XY, as the corresponding author, designed the protocol and revised the manuscript. All authors read and approved the final manuscript.

\section{Ethics approval and consent to participate}

Not applicable.

\section{Patient consent for publication}

Not applicable.

\section{Competing interests}

The authors declare that they have no competing interests.

\section{References}

1. Siegel RL, Miller KD and Jemal A: Cancer statistics, 2015. CA Cancer J Clin 65: 5-29, 2015.

2. Neville BW and Day TA: Oral cancer and precancerous lesions. CA Cancer J Clin 52: 195-215, 2002.

3. Fusco A and Fedele M: Roles of HMGA proteins in cancer. Nat Rev Cancer 7: 899-910, 2007

4. Quan J, Johnson NW, Zhou G, Parsons PG, Boyle GM and Gao J: Potential molecular targets for inhibiting bone invasion by oral squamous cell carcinoma: A review of mechanisms. Cancer Metastasis Rev 31: 209-219, 2012.

5. Bella L, Zona S, Nestal de Moraes G and Lam EW: FOXM1: A key oncofoetal transcription factor in health and disease. Semin Cancer Biol 29: 32-39, 2014.

6. Wang Z, Ahmad A, Li Y, Banerjee S, Kong D and Sarkar FH: Forkhead box M1 transcription factor: A novel target for cancer therapy. Cancer Treat Rev 36: 151-156, 2010.

7. Halasi M and Gartel AL: FOX(M1) news-it is cancer. Mol Cancer Ther 12: 245-254, 2013.

8. Leung TW, Lin SS, Tsang AC, Tong CS, Ching JC, Leung WY, Gimlich R, Wong GG and Yao KM: Over-expression of FoxM1 stimulates cyclin B1 expression. FEBS Lett 507: 59-66, 2001.

9. Shi M, Cui J and Xie K: Signaling of miRNAs-FOXM1 in cancer and potential targeted therapy. Curr Drug Targets 14: 1192-1202, 2013.

10. Zhang HG, Xu XW, Shi XP, Han BW, Li ZH, Ren WH, Chen PJ, Lou YF, Li B and Luo XY: Overexpression of forkhead box protein M1 (FOXM1) plays a critical role in colorectal cancer. Clin Transl Oncol 18: 527-532, 2016.

11. Livak KJ and Schmittgen TD: Analysis of relative gene expression data using real-time quantitative PCR and the 2(-Delta Delta C(T)) method. Methods 25: 402-408, 2001.

12. Dull T, Zufferey R, Kelly M, Mandel RJ, Nguyen M, Trono D and Naldini L: A third-generation lentivirus vector with a conditional packaging system. J Virol 72: 8463-8471, 1998.

13. Korver W, Schilham MW, Moerer P, van den Hoff MJ, Dam K, Lamers WH, Medema RH and Clevers H: Uncoupling of S phase and mitosis in cardiomyocytes and hepatocytes lacking the winged-helix transcription factor Trident. Curr Biol 8: $1327-1330,1998$.

14. Jin H, Li XJ, Park MH and Kim SM: FOXM1-mediated downregulation of UPA and MMP9 by 3,3'-diindolylmethane inhibits migration and invasion of human colorectal cancer cells. Oncol Rep 33: 3171-3177, 2015. 
15. Quan M, Wang P, Cui J, Gao Y and Xie K: The roles of FOXM1 in pancreatic stem cells and carcinogenesis. Mol Cancer 12: 159, 2013.

16. Zhu H: Forkhead box transcription factors in embryonic heart development and congenital heart disease. Life Sci 144: 194-201, 2016.

17. Gormally MV, Dexheimer TS, Marsico G, Sanders DA, Lowe C Matak-Vinković D, Michael S, Jadhav A, Rai G, Maloney DJ, et al: Suppression of the FOXM1 transcriptional programme via novel small molecule inhibition. Nat Commun 5: 5165, 2014.

18. Chu XY, Zhu ZM, Chen LB, Wang JH, Su QS, Yang JR, Lin Y, Xue LJ, Liu XB and Mo XB: FOXM1 expression correlates with tumor invasion and a poor prognosis of colorectal cancer. Acta Histochem 114: 755-762, 2012.

19. Rao DD, Wang Z, Senzer N and Nemunaitis J: RNA interference and personalized cancer therapy. Discov Med 15: 101-110, 2013.

20. Swanton C, Nicke B and Downward J: RNA interference, DNA methylation, and gene silencing: A bright future for cancer therapy? Lancet Oncol 5: 653-654, 2004.

21. Wang Z, Rao DD, Senzer N and Nemunaitis J: RNA interference and cancer therapy. Pharm Res 28: 2983-2995, 2011.
22. Root DE, Hacohen N, Hahn WC, Lander ES and Sabatini DM: Genome-scale loss-of-function screening with a lentiviral RNAi library. Nat Methods 3: 715-719, 2006.

23. Kalin TV, Ustiyan V and Kalinichenko VV: Multiple faces of FoxM1 transcription factor: Lessons from transgenic mouse models. Cell Cycle 10: 396-405, 2011.

24. Wakino S, Kintscher U, Kim S, Yin F, Hsueh WA and Law RE: Peroxisome proliferator-activated receptor gamma ligands inhibit retinoblastoma phosphorylation and G1 $>$ S transition in vascular smooth muscle cells. J Biol Chem 275: 22435-22441, 2000.

25. Liu M, Dai B, Kang SH, Ban K, Huang FJ, Lang FF, Aldape KD, Xie TX, Pelloski CE, Xie K, et al: FoxM1B is overexpressed in human glioblastomas and critically regulates the tumorigenicity of glioma cells. Cancer Res 66: 3593-3602, 2006.

26. Rubin MA: Insights into the mechanism of organ-specific cancer metastasis. Cancer Discov 4: 1262-1264, 2014.

27. Kessenbrock K, Wang CY and Werb Z: Matrix metalloproteinases in stem cell regulation and cancer. Matrix Biol 44-46: 184-190, 2015. 